\title{
Scopes and limits of modality in quantum mechanics
}

\author{
Graciela Domenech ${ }^{* 1,3}$, Hector Freytes ${ }^{2}$ and Christian de Ronde ${ }^{3,4}$ \\ July 19, 2018
}

\author{
1. Instituto de Astronomía y Física del Espacio (IAFE) \\ Casilla de Correo 67, Sucursal 28, 1428 Buenos Aires, Argentina \\ 2. Dipartimento di Scienze e Pedagogiche e Filosofiche - Universita degli Studi di Cagliari \\ Via Is Mirrionis 1, 09123, Cagliari - Italia \\ 3. Center Leo Apostel (CLEA) \\ 4. Foundations of the Exact Sciences (FUND) \\ Brussels Free University - Krijgskundestraat 33, 1160 Brussels - Belgium
}

\begin{abstract}
We develop an algebraic frame for the simultaneous treatment of actual and possible properties of quantum systems. We show that, in spite of the fact that the language is enriched with the addition of a modal operator to the orthomodular structure, contextuality remains a central feature of quantum systems.
\end{abstract}

\section{Introduction}

Contextuality is one of the main features of the discourse about quantum systems and has been studied from different approaches. We are interested here in algebraic versions related to partial valuations of the orthomodular lattice of closed subspaces of Hilbert space. This proposal allows to identify the constraints imposed by the structure to the relation between actuality and possibility and the discourse that includes both type of propositions.

The paper is organized as follows: in Sec. 2 we discuss the contextual character of quantum systems from an algebraic perspective. Sec. 3 introduces the desiderata of modal interpretations of quantum mechanics involved in our treatment. We devote Sec. 4 to expose the algebraic structure which represents the orthomodular lattice enriched with modal operators. In Sec. 5 we show how the discourse about properties is genuinely enlarged giving an adequate framework to represent the Born rule. In Sec. 6 we use Kochen-Specker theorem to prove that the contextual character of quantum mechanics is maintained even when the discourse is enriched with modalities. Finally, we outline our conclusions.

\section{Contextuality in quantum systems}

In paper [9], we dealt with the problem of the limits imposed by the orthomodular structure of projection operators to the possibility of thinking of properties possessed by an isolated quantum system. This is an important point in the discussion about quantum systems because almost every problem in the relation between the mathematical formalism and what may be called our experience about the behavior of physical objects can be encoded in the question about the possible meaning of the proposition the physical magnitude $\mathcal{A}$ has a value and the value is this or that real number. Already from the first formalizations, this point was recognized. For example, P.A.M. Dirac stated in his famous book: "The expression that an observable 'has a particular value' for a particular state is permissible in quantum mechanics in the special case when a measurement of the observable is certain to lead to the particular value, so that the state is an eigenstate of the observable. It may easily be verified from the algebra

*Fellow of the Consejo Nacional de Investigaciones Científicas y Técnicas (CONICET) 
that, with this restricted meaning for an observable 'having a value', if two observables have values for a particular state, then for this state the sum of the two observables (if the sum is an observable) has a value equal to the sum of the values of the two observables separately and the product of the two observables (if this product is an observable) has a value equal to the product of the values of the two observables separately" [7. This last point is the requirement of the functional compatibility condition (FUNC), to which we will return later. As long as we limit ourselves to speaking about measuring results and avoid being concerned with what happens to nature when she is not measured, quantum mechanics carries out predictions with great accuracy. But if we naively try to interpret eigenvalues as actual values of the physical properties of a system, we are faced with all kind of no-go theorems that preclude this possibility. Most remarkable is the Kochen-Specker (KS) theorem that rules out the non-contextual assignment of values to physical magnitudes [17]. In [9] we gave algebraic, topological and categorial versions of the KS theorem. To include modalities in our analysis we will deal with the algebraic form in terms of valuations. So we recall the main features of our discussion there.

Let $\mathcal{H}$ be the Hilbert space associated with the physical system and $L(\mathcal{H})$ be the set of closed subspaces on $\mathcal{H}$. If we consider the set of these subspaces ordered by inclusion, then $L(\mathcal{H})$ is a complete orthomodular lattice [18. It is well known that each self-adjoint operator $\mathbf{A}$ representing a physical magnitude $\mathcal{A}$ has associated a Boolean sublattice $W_{A}$ of $L(\mathcal{H})$. More precisely, $W_{A}$ is the Boolean algebra of projectors $\mathbf{P}_{i}$ of the spectral decomposition $\mathbf{A}=\sum_{i} a_{i} \mathbf{P}_{i}$. We will refer to $W_{A}$ as the spectral algebra of the operator A. Any proposition about the system is represented by an element of $L(\mathcal{H})$ which is the algebra of quantum logic introduced by G. Birkhoff and J. von Neumann [2].

Assigning values to a physical quantity $\mathcal{A}$ is equivalent to establishing a Boolean homomorphism $v: W_{A} \rightarrow \mathbf{2} 13$, being $\mathbf{2}$ the two elements Boolean algebra. So it is natural to consider the following definition:

Definition 2.1 Let $\left(W_{i}\right)_{i \in I}$ be the family of Boolean sublattices of $L(\mathcal{H})$. A global valuation over L $(\mathcal{H})$ is a family of Boolean homomorphisms $\left(v_{i}: W_{i} \rightarrow \mathbf{2}\right)_{i \in I}$ such that $v_{i}\left|W_{i} \cap W_{j}=v_{j}\right| W_{i} \cap W_{j}$ for each $i, j \in I$

Were it possible, this global valuation would give the values of all magnitudes at the same time maintaining a compatibility condition in the sense that whenever two magnitudes share one or more projectors, the values assigned to those projectors are the same from every context.

But the KS theorem assures that we cannot assign real numbers pertaining to their spectra to operators A in such a way to satisfy the functional composition principle (FUNC) which is the expression of the "natural" requirement mentioned by Dirac that, for any operator A representing a dynamical variable and any real-valued function $f(\mathbf{A})$, the value of $f(\mathbf{A})$ is the corresponding function of the value of $\mathbf{A}$. In the algebraic terms of the previous definition, the KS theorem reads:

Theorem 2.2 If $\mathcal{H}$ is a Hilbert space such that $\operatorname{dim}(\mathcal{H})>2$, then a global valuation over $L(\mathcal{H})$ is not possible.

Of course contextual valuations allow us to refer to different sets of actual properties of the system which define its state in each case. Algebraically, a contextual valuation is a Boolean valuation over one chosen spectral algebra. In classical particle mechanics it is possible to define a Boolean valuation of all propositions, that is to say, it is possible to give a value to all the properties in such a way of satisfying FUNC. This possibility is lost in the quantum case.

Now we intend to show how this discussion is able to include modalities, i.e. to consider also possibility and necessity of propositions about the properties of physical systems: what may be the case (what the possible physical situations are) and what is necessarily the case. The consideration of possibility is of course always present in quantum theories. What we propose here is an algebraic consideration of this type of sentences and their articulation with those about actual properties. Several attempts to obtain modal extensions of the orthomodular systems are found in the literature. One possibility developed in 8 allows to embed orthomodular propositional systems in modal systems. Another extension is provided by adding quantifiers to the orthomodular structure [14, 15], so generalizing the monadic extension of the Boolean algebras [12]. In our case, we enrich the orthomodular structure with a modal operator thus obtaining an algebraic variety such that each orthomodular lattice can be represented by an algebra of this variety. On the other hand, this operator acts as a quantifier in the sense of [14, 15]. The physical 
motivation for this construction is the purpose to link consistently the propositions about actual and possible properties of the system in a single structure.

\section{Modal interpretations}

Modal interpretations of quantum mechanics [21, 4, 5, 6, face the problem of finding an objective reading of the accepted mathematical formalism of the theory, a reading "in terms of properties possessed by physical systems, independently of consciousness and measurements (in the sense of human interventions)" [6]. These interpretations intend to consistently include the possible properties of the system in the discourse and so find a new link between the state of the system and the probabilistic character of its properties, namely, sustaining that the interpretation of the quantum state must contain a modal aspect. The name modal interpretation was for the first time used by B. van Fraassen 20 following modal logic, precisely the logic that deals with possibility and necessity. The fundamental point is the purpose of interpreting "the formalism as providing information about properties of physical systems" [6]. In this context, a physical property of a system is "a definite value of a physical quantity belonging to this system; i.e., a feature of physical reality" [6. As usual, definite values of physical magnitudes correspond to yes/no propositions represented by orthogonal projection operators acting on vectors belonging to the Hilbert space of the (pure) states of the system [16].

Modal interpretations may be thought to be a study of the constraints under which one is able to talk a consistent classical discourse without contradiction with the quantum formalism. To study this issue and in order to avoid inconsistencies, we face the problem of modalities in the frame of algebraic logic. To do so, we build a variety that is an expansion of the orthomodular lattices by adding an operator, the possibility operator, to these structures. It will represent the possibility of occurrence of a property, measurable in terms of the Born rule. The analysis of the changes introduced by allowing modalities will be performed, as in 9], for the case of pure states. In spite of the restrictions this imposes to the comparison with the general case of modal interpretations, we think it contributes to enlighten the discussion all the same. In a following step, we will extend the treatment to the factorized space of subsystems.

\section{An algebraic study of modality}

First we recall from [3, 18, 1, some notions of the universal algebra and lattice theory that will play an important role in what follows. For each algebra $A$, we denote by $\operatorname{Con}(A)$, the congruence lattice of $A$, the diagonal congruence is denoted by $\Delta$ and the largest congruence $A^{2}$ is denoted by $\nabla$. $\theta$ is called factor congruence iff there is a congruence $\theta^{*}$ on $A$ such that, $\theta \wedge \theta^{*}=\Delta, \theta \vee \theta^{*}=\nabla$ and $\theta$ permutes with $\theta^{*}$. If $\theta$ and $\theta^{*}$ is a pair of factor congruences on $A$ then $A \cong A / \theta \times A / \theta^{*}$. $A$ is directly indecomposable if $A$ is not isomorphic to a product of two non trivial algebras or, equivalently $\Delta, \nabla$ are the only factor congruences in $A$. We say that $A$ is subdirect product of a family of $\left(A_{i}\right)_{i \in I}$ of algebras if there exists an embedding $f: A \rightarrow \prod_{i \in I} A_{i}$ such that $\pi_{i} f: A \rightarrow A_{i}$ is a surjective homomorphism for each $i \in I$ where $\pi_{i}$ is the projection onto $A_{i} . A$ is subdirectly irreducible iff $A$ is trivial or there is a minimum congruence in $\operatorname{Con}(A)-\Delta$. It is clear that a subdirectly irreducible algebra is directly indecomposable. An important result due to Birkhoff is that every algebra $A$ is subdirect product of subdirectly irreducibles algebras. In a Boolean algebra $A$, congruences are identifiable to certain subsets called filters. $F \subset A$ is a filter iff it satisfies: if $a \in F$ and $a \leq x$ then $x \in F$ and if $a, b \in F$ then $a \wedge b \in F$. F is a proper filter iff $F \neq A$ or, equivalently, $0 \notin F$. If $X \subseteq A$, the filter $F_{X}$ generated by $X$ is the minimum filter containing $X$. A proper filter $F$ is maximal iff the quotient algebra $A / F$ is isomorphic to $\mathbf{2}$. It is well known that each proper filter can be extended to a maximal one.

We denote by $\mathcal{O} \mathcal{M L}$ the variety of orthomodular lattices. Let $L=\langle L, \vee, \wedge, \neg, 0,1\rangle$ be an orthomodular lattice. Given $a, b, c$ in $L$, we write: $(a, b, c) D$ iff $(a \vee b) \wedge c=(a \wedge c) \vee(b \wedge c) ;(a, b, c) D^{*}$ iff $(a \wedge b) \vee c=$ $(a \vee c) \wedge(b \vee c)$ and $(a, b, c) T$ iff $(a, b, c) D,(\mathrm{a}, \mathrm{b}, \mathrm{c}) D^{*}$ hold for all permutations of $a, b, c$. An element $z$ of a lattice $L$ is called a central iff for all elements $a, b \in L$ we have $(a, b, z) T$. We denote by $Z(L)$ the set of all central elements of $L$ and it is called the center of $L . Z(L)$ is a Boolean sublattice of $L$ [18, Theorem $4.15]$.

Proposition 4.1 [18, Lemma 29.9 and Lemma 29.16] Let $L$ be an orthomodular lattice then we have 
1. $z \in Z(L)$ if and only if $a=(a \wedge z) \vee(a \wedge \neg z)$ for each $a \in L$

2. If $L$ is complete then $Z(L)$ is a complete lattice and for each family $\left(z_{i}\right)_{i}$ in $Z(L)$ and a $\in L$, $a \wedge \bigvee z_{i}=\bigvee\left(a \wedge Z_{i}\right)$

Factor congruences in $L$ are identifiable to the elements of the center $Z(L)$. More precisely if $z \in$ $Z(L)$, the binary relation $\Theta_{z}$ on $A$ defined by $a \Theta_{z} b$ iff $a \wedge z=b \wedge z$ is a congruence on $L$, such that $L \cong L / \Theta_{z} \times L / \Theta_{\neg z}$.

Now we build up a framework to include modal propositions in the same structure as actual ones. To do so, we enrich the orthomodular lattice with a modal operator taking into account the following considerations:

1. Propositions about the properties of the physical system will be interpreted in the orthomodular lattice of subspaces of the Hilbert space of the (pure) states of the system. Thus we will retain this structure in our extension.

2. Given a proposition about the system, it is possible to define a context from which one can predicate with certainty about it (and about a set of propositions that are compatible with it) and predicate probabilities about the other ones. This is to say that one may predicate truth or falsity of all possibilities at the same time, i.e. possibilities allow an interpretation in a Boolean algebra. In rigorous terms, for each proposition $P$, if we refer with $\diamond P$ to the possibility of $P$, then $\diamond P$ will be a central element of the orthomodular structure.

3. If $P$ is a proposition about the system and $P$ occurs, then it is trivially possible that $P$ occurs. This is expressed as $P \leq \diamond P$.

4. To assume an actual property and a complete set of properties that are compatible with it determines a context in which the classical discourse holds. Classical consequences that are compatible with it, for example probability asignements to the actuality of other propositions, share the classical frame. These consequences are the same ones as those which would be obtained by considering the original actual property as a possible one. This is interpreted as, if $P$ is a property of the system, $\diamond P$ is the smallest central element greater than $P$.

The algebraic study will be performed using the necessity operator $\square$ instead of the possibility one $\diamond$ because of technical reasons. Then it will be possible to define the possibility operator from the necessity one.

Definition 4.2 Let $A$ be an orthomodular lattice. We say that $A$ is Boolean saturated iff for each $a \in A$ the set $\{z \in Z(A): z \leq a\}$ has a maximum. In this case such maximum is denoted by $\square(a)$.

Example 4.3 In view of Proposition 4.1, orthomodular complete lattices with $\square(a)=\bigvee\{z \in Z(L)$ : $z \leq a\}$ as an operator, are examples of Boolean saturated orthomodular lattices.

Proposition 4.4 Let $A$ be an orthomodular lattice. Then $A$ is Boolean saturated iff there exists an unary operator $\square$ acting on the elements of $A$ satisfying

$$
\begin{aligned}
& \text { S1 } \square x \leq x \\
& \text { S2 } \square 1=1 \\
& \text { S3 } \square \square x=\square x \\
& \text { S4 } \square(x \wedge y)=\square(x) \wedge \square(y) \\
& \text { S5 } y=(y \wedge \square x) \vee(y \wedge \neg \square x) \\
& \text { S6 } \square(x \vee \square y)=\square x \vee \square y
\end{aligned}
$$




$$
S 7 \square(\neg x \vee(y \wedge x)) \leq \neg \square x \vee \square y
$$

Proof: $\quad$ Suppose that $A$ is Boolean saturated. S1), S2) and S3) are trivial. S4) Since $x \wedge y \leq x$ and $x \wedge y \leq y$ then $\square(x \wedge y) \leq \square(x) \wedge \square(y)$. For the converse, $\square(x) \leq x$ and $\square(y) \leq y$, thus $\square(x) \wedge \square(y) \leq \square(x \wedge y)$. S5) Follows from Proposition 4.1 since $\square(x) \in Z(A)$. S6) For simplicity let $z=\square y$. From the precedent item and taking into account that $z \in Z(L)$ we have that $\square(z \vee x) \wedge \square(\neg z \vee x)=\square((z \vee x) \wedge(\neg z \vee x))=\square(x)$. Since $\neg z \leq \square(\neg z \vee x)$ then we have that $1=z \vee \neg z \leq z \vee \square(\neg z \vee x)$. Also we have $z \leq \square(z \vee x)$. Finally $z \vee \square(x)=(z \vee \square(z \vee x)) \wedge(z \vee \square(\neg z \vee x))=$ $(z \vee \square(z \vee x)) \wedge 1=\square(z \vee x)$ i.e. $\square(x \vee \square y)=\square x \vee \square y$. S7) Since $\square(x) \leq x$ then $\neg x \leq \neg \square x$, we have that $\neg x \vee(y \wedge x) \leq \neg \square x \vee y$. Using the precedent item $\square(\neg x \vee(y \wedge x)) \leq \square(\neg \square x \vee y)=\neg \square x \vee \square y$ since $\neg \square x \in Z(A)$.

For the converse, let $a \in A$ and $\{z \in Z(A): z \leq a\}$. By $S 1$ and $S 5$ it is clear that $\square a \in\{z \in Z(A)$ : $z \leq a\}$. We see that $\square a$ is the upper bound of the set. Let $z \in Z(A)$ such that $z \leq a$ then $1=\neg z \vee(a \wedge z)$. Using $S 2$ and $S 7$ we have $1=\square 1=\square(\neg z \vee(a \wedge z)) \leq \neg \square z \vee a=\neg z \vee a$. Therefore $z=z \wedge(\neg z \vee \square a)$ and since $z$ is central $z=z \wedge \square a$ resulting $z \leq \square a$. Finally $\square a=\operatorname{Max}\{z \in Z(A): z \leq a\}$.

Theorem 4.5 The class of Boolean saturated orthomodular lattices constitutes a variety which is axiomatized by

1. Axioms of $\mathcal{O M L}$

2. $S 1, \ldots, S 7$

Proof: $\quad$ Obvious by Proposition 4.4

Boolean saturated orthomodular lattices are algebras $\langle A, \wedge, \vee, \neg, \square, 0,1\rangle$ of type $\langle 2,2,1,1,0,0\rangle$ and the variety they constitute will be noted as $\mathcal{O} \mathcal{M L}^{\square}$.

On each algebra of $\mathcal{O} \mathcal{M L}^{\square}$ we can define the possibility operator as unary operation $\diamond$ given by

$$
\diamond x=\neg \square \neg x
$$

Proposition 4.6 Let $A$ be a Boolean saturated orthomodular lattice and $a, b \in A$. Then we have

1. $a \leq \diamond a$

2. $\diamond a=\operatorname{Min}\{z \in Z(A): a \leq z\}$

Proof: $\quad$ We first note that $\diamond a \in Z(A)$ since $\square \neg a \in Z(A)$. On the other hand $\square \neg a \leq \neg a$ and then $a=\neg \neg a \leq \neg \square \neg a=\diamond a$. If $z \in Z(A)$ such that $a \leq z$ then $\neg z \leq \neg a$ resulting $\neg z \leq \square \neg a$. Thus $\diamond a=\neg \square \neg a \leq z$.

Remark 4.7 From 4.6 it may be seen that Boolean saturated orthomodular lattices satisfy the four items which motivate our approach.

Theorem 4.8 Let $L$ be an orthomodular lattice. Then there exists an orthomodular monomorphism $f: L \rightarrow L^{\square}$ such that $L^{\square} \in \mathcal{O M}^{\square}$.

Proof: $\quad$ Let $f: L \rightarrow \prod_{i \in I} L_{i}$ be a subdirect embedding of $L$. Since $L_{i}$ is subdirectly irreducible then $Z\left(L_{i}\right)=\{0,1\}$ for each $i \in I$ resulting $Z\left(\prod_{i \in I} L_{i}\right)$ a complete Boolean algebra and so $\prod_{i \in I} L_{i}$ is Boolean saturated.

In this case we may say that each orthomodular lattice can be represented by a Boolean saturated one. In this sense, the embedding of orthomodular systems in modal systems proposed in [8] is maintained. On the other hand, we see that the defined modal operators are quantifiers in the sense of [14, 15].

For each orthomodular lattice $L$, if $f: L \rightarrow L^{\square}$ such that $L^{\square} \in \mathcal{O} \mathcal{M} \mathcal{L}^{\square}$ is an orthomodular monomorphism, we refer to $L^{\square}$ as a modal extension of $L$. In this case, we may see the lattice $L$ as a subset of $L^{\square}$. 


\section{Modalities: enlargement of the expressivity of the discourse}

It is clear that the addition of modalities gives by itself greater expressive power to the language of propositions about the system. But what we want to emphasize is that it gives an adequate framework to represent, for example, the Born rule for the probability of actualization of a property, something that has no place in the orthomodular lattice alone. In order to develop these ideas, we need to prove which conditions on elements of a subset $A$ are necessary to make $\langle A\rangle_{L}$, the sublattice generated by $A$, a Boolean sublattice.

Definition 5.1 Let $L$ be an orthomodular lattice and $a, b \in L$. Then $a$ commutes with $b$ if and only if $a=(a \wedge b) \vee(a \wedge \neg b)$. A non-empty subset $A$ is called a Greechie set iff for any three different elements of $A$, at least one commutes with the other two.

Proposition 5.2 Let $L$ be an orthomodular lattice. If $A$ is a Greechie set in $L$ such that for each $a \in A, \neg a \in A$ then, $\langle A\rangle_{L}$ is Boolean sublattice.

Proof: It is well known from [11] that $\langle A\rangle_{L}$ is a distributive sublattice of $L$. Since distributive orthomodular lattices are Boolean algebras, we only need to see that $\langle A\rangle_{L}$ is closed by $\neg$. To do that we use induction on the complexity of terms of the subuniverse generated by $A$. For $\operatorname{comp}(a)=0$, it follows from the fact that $A$ is closed by negation. Assume validity for terms of the complexity less than $n$. Let $\tau$ be a term such that $\operatorname{comp}(\tau)=n$. If $\tau=\neg \tau_{1}$ then $\neg \tau \in\langle A\rangle_{L}$ since $\neg \tau=\neg \neg \tau_{1}=\tau_{1}$ and $\tau_{1} \in\langle A\rangle_{L}$. If $\tau=\tau_{1} \wedge \tau_{2}, \neg \tau=\neg \tau_{1} \vee \neg \tau_{2}$. Since $\operatorname{comp}\left(\tau_{i}\right)<n, \neg \tau_{i} \in\langle A\rangle_{L}$ for $i=1,2$ resulting $\neg \tau \in\langle A\rangle_{L}$. We use the same argument in the case $\tau=\tau_{1} \vee \tau_{2}$. Finally $\langle A\rangle_{L}$ is a Boolean sublattice.

Definition 5.3 Let $L$ be an orthomodular lattice and $L^{\square} \in \mathcal{O} \mathcal{M L}^{\square}$ be a modal extension of $L$. We define the possibility space of $L$ in $L^{\square}$ as

$$
\diamond L=\langle\{\diamond p: p \in L\}\rangle_{L^{\square}}
$$

The possibility space represents the modal content added to the discourse about properties of the system.

Proposition 5.4 Let $L$ be an orthomodular lattice, $W$ a Boolean sublattice of $L$ and $L^{\square} \in \mathcal{O M}^{\square}$ a modal extension of $L$. Then $\langle W \cup \diamond L\rangle_{L^{\square}}$ is a Boolean sublattice of $L^{\square}$. In particular $\diamond L$ is a Boolean sublattice of $Z\left(L^{\square}\right)$.

Proof: $\quad$ Follows from Proposition 5.2 since $W \cup \diamond L$ is a Greechie set closed by $\neg$.

We know that, in the orthomodular lattice of the properties of the system, it is always possible to choose a context in which any possible property pertaining to this context can be considered as an actual property. We formalize this fact in the following definition and then we prove that this is always possible in our modal structure.

Definition 5.5 Let $L$ be an orthomodular lattice, $W$ a Boolean sublattice of $L, p \in W$ and $L^{\square}$ be a modal extension of $L$. If $f: \diamond L \rightarrow \mathbf{2}$ is a Boolean homomorphism such that $f(\diamond p)=1$ then an actualization of $p$ compatible with $f$ is a Boolean homomorphism $f_{p}: W \rightarrow \mathbf{2}$ such that

1. $f_{p}(p)=1$

2. There exists a Boolean homomorphism $g:\langle W \cup \diamond L\rangle_{L^{\square}} \rightarrow \mathbf{2}$ such that $g \mid W=f_{p}$ and $g \mid \diamond L=f$

Theorem 5.6 Let $L$ be an orthomodular lattice, $W$ a Boolean sublattice of $L, p \in W$ and $L^{\square}$ be a modal extension of $L$. If $f: \diamond L \rightarrow \mathbf{2}$ is a Boolean homomorphism such that $f(\diamond p)=1$ then there exists an actualization of $p$ compatible with $f$.

Proof: $\quad$ Let $F$ be the filter associated with the Boolean homomorphism $f$. We consider the $\langle W \cup \diamond L\rangle_{L^{\square-}}$ filter $F_{p}$ generated by $F \cup\{p\}$. We want to see that $F_{p}$ is a proper filter. If $F_{p}$ is not proper, then there exists $a \in F$ such that $a \wedge p \leq 0$. Thus $p \leq \neg a$ being $\neg a$ a central element. But $\diamond p$ is the smallest Boolean element greater than $p$, then $\diamond p \leq \neg a$ or equivalently $\diamond p \wedge a=0$ And this is a contradiction since 
$\diamond p, a \in F$ being $F$ a proper filter. Thus we may extend $F_{p}$ to be a maximal filter $F_{M}$ in $\langle W \cup \diamond L\rangle_{L^{\square}}$, resulting the natural projection $\langle W \cup \diamond L\rangle_{L^{\square}} \rightarrow\langle W \cup \diamond L\rangle_{L^{\square}} / F_{M} \approx \mathbf{2}$ an actualization of $p$ compatible with $f$.

The next theorem allows an algebraic representation of the Born rule which quantifies possibilities from a chosen spectral algebra.

Theorem 5.7 Let $L$ be an orthomodular lattice, $W$ a Boolean sublattice of $L$ and $f: W \rightarrow \mathbf{2}$ a Boolean homomorphism. If we consider a modal extension $L^{\square}$ of $L$ then there exists a Boolean homomorphism $f^{*}:\langle W \cup \diamond L\rangle_{L^{\square}} \rightarrow \mathbf{2}$ such that $f^{*} \mid W=f$.

Proof: $\quad$ Let $i: W \rightarrow\langle W \cup \diamond L\rangle_{L^{\square}}$ be the Boolean canonical embedding. If we consider the following diagram:

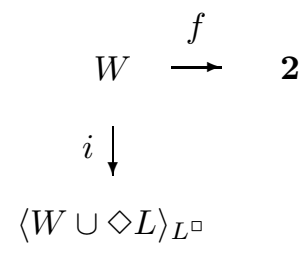

we see that there exists a Boolean homomorphism $f^{*}:\langle W \cup \diamond L\rangle_{L^{\square}} \rightarrow \mathbf{2}$ such that $f^{*} \mid W_{A}=f$ because 2 is injective in the variety of Boolean algebras [19].

We note that this reading of the Born rule is a kind of converse of the possibility of actualizing properties given by Theorem 5.6

\section{Kochen-Specker theorem: a limit also for modalities}

The addition of modalities to the discourse about the properties of a quantum system enlarges its expressive power. At first sight it may be thought that this could help to circumvent contextuality, allowing to refer to physical properties belonging to the system in an objective way that resembles the classical picture. But this is not the case as we have announced in [10. To prove it here, we introduce an algebraic representation of the notion of global actualization:

Definition 6.1 Let $L$ be an orthomodular lattice, $\left(W_{i}\right)_{i \in I}$ the family of Boolean sublattices of $L$ and $L^{\square}$ a modal extension of $L$. If $f: \diamond L \rightarrow \mathbf{2}$ is a Boolean homomorphism, an actualization compatible with $f$ is a global valuation $\left(v_{i}: W_{i} \rightarrow \mathbf{2}\right)_{i \in I}$ such that $v_{i}\left|W_{i} \cap \diamond L=f\right| W_{i} \cap \diamond L$ for each $i \in I$.

Compatible actualizations represent the passage from possibility to actuality.

Theorem 6.2 Let $L$ be an orthomodular lattice. Then $L$ admits a global valuation iff for each possibility space there exists a Boolean homomorphism $f: \diamond L \rightarrow \mathbf{2}$ that admits a compatible actualization.

Proof: $\quad$ Suppose that $L$ admits a global valuation $\left(v_{i}: W_{i} \rightarrow \mathbf{2}\right)_{i \in I}$. Let $L^{\square}$ be a modal extension of $L$ and consider $A_{i}=W_{i} \cap \diamond L$. Let $f_{0}=\bigcup_{i} A_{i} \rightarrow \mathbf{2}$ such that $f_{0}(x)=v_{i}(x)$ if $x \in W_{i} . f_{0}$ is well defined since $\left(v_{i}\right)_{i}$ is a global valuation. If we consider $\left\langle\bigcup_{i} A_{i}\right\rangle_{L^{\square}}$, the subalgebra of $L^{\square}$ generated by the join of the family $\left(A_{i}\right)$, it may be proved that it is a Boolean subalgebra of the possibility space $\diamond L$. We can extended $f_{0}$ to a Boolean homomorphism $f_{0}^{*}:\left\langle\bigcup_{i} A_{i}\right\rangle_{L^{\square}} \rightarrow \mathbf{2}$. Since $\mathbf{2}$ is injective in the variety of Boolean algebras [19], there exits a Boolean homomorphism $f: \diamond L \rightarrow \mathbf{2}$ such that the following diagram is commutative

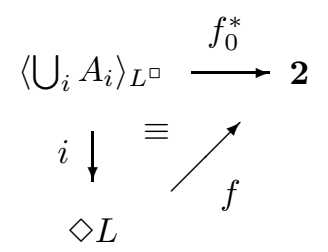


Thus $f: \diamond L \rightarrow \mathbf{2}$ admits a compatible actualization. The converse is immediate.

Since the possibility space is a Boolean algebra, there exists a Boolean valuation of the possible properties. But in view of the last theorem, an actualization that would correspond to a family of compatible global valuations is prohibited. Thus the theorem states that the contextual character of quantum mechanics is maintained even when the discourse is enriched with modalities.

\section{Conclusions}

From the algebraic characterization of contextuality given by the non existence of compatible global valuations over the orthomodular structure, we show that, if this structure is enriched with modal operators, the discourse about properties is genuinely enlarged. However, the contextual character of the complete language is maintained. Thus contextuality remains a main feature of quantum systems even when modalities are taken into account.

\section{Acknowledgements}

This work was partially supported by the following grants: PICT 04-17687 (ANPCyT), PIP No 1478/01 (CONICET), UBACyT No X081 and X204. G. D. is fellow of the Consejo Nacional de Investigaciones Científicas y Técnicas (CONICET).

\section{References}

[1] Balbes, R. and Dwinger, Ph. Distribuive Lattices, (University of Missouri Press, Columbia, MO, 1974).

[2] Birkhoff, G. and von Neuman, J., "The logic of quantum mechanics", Ann. Math. 27, 823-843 (1936).

[3] Burris, S. and Sankappanavar, H. P., A Course in Universal Algebra, (Graduate Text in Mathematics, Vol. 78, Springer-Verlag, New York Heidelberg Berlin, 1981).

[4] Dieks, D., "The formalism of quantum theory: an objective description of reality", Annalen der Physik 7, 174-190 (1988).

[5] Dieks, D., "Quantum mechanics without the projection postulate and its realistic interpretation", Found. Phys. 19, 1397-1423 (1989).

[6] Dieks, D., "Quantum mechanics: an intelligible description of objective reality?", Found. Phys. 35, 399-415 (2005).

[7] Dirac, P.A.M. The principle of quantum mechanics, (Oxford University Press, 1958)

[8] Dishkant, H., "Imbedding of the quantum logic in the modal system of Brower", J. Symb. Logic 42, 321-328 (1978).

[9] Domenech, G. and Freytes, H., "Contextual logic for quantum systems", J. Math. Phys. 46, 012102 (2004).

[10] Domenech, G., Freytes, H. and de Ronde, C., "Kochen-Specker theorem: a limit also for modalities", submitted to Found. Phys. Lett. (2006).

[11] Greechie, R., "On generating distributive sublattices of orthomodular lattices", Proc. Amer. math. Soc. 67, 17-22 (1977).

[12] Halmos, P., "Algebraic logic I, monadic Boolean algebras", Compositio Math. 12, 217-249 (1955).

[13] Isham, C. and Butterfield, J., "A topos perspective on the Kochen-Specker theorem I", Int. J. Theor. Phys. 37, 2669-2773 (1998). 
[14] Janowitz, M. F., "Quantifiers and orthomodular lattices", Pac. J. Math. 13, 1241-1249 (1963).

[15] Janowitz, M. F., "Quantifier theory on quasi-orthomodular lattices", Ill. J. Math. 9, 660-676 (1965).

[16] Jauch, J. M., Foundations of Quantum Mechanics, (Addison-Wesley Pub. Co., Massachusetts, 1966).

[17] Kochen, S. and Specker, E. P. "The problem of hidden variables in quantum mechanics", J. Math. Mech. 17, 9-87 (1967).

[18] Maeda, F. and Maeda, S., Theory of Symmetric Lattices, (Springer-Verlag, Berlin, 1970).

[19] Sikorski, R., "A theorem on extensions of homomorphism", Ann. Soc. Pol. Math. , 21, 332-335 (1948).

[20] van Fraassen, B. C., "A modal interpretation of quantum mechanics", in Current issues in quantum logic, Beltrametti E. G. and van Fraassen, B. C., eds., (Plenum Press, New York, 229-258, 1981).

[21] van Fraassen, B. C., Quantum Mechanics: An Empiricist View, (Clarendon, Oxford, 1991). 